\title{
Une stabilisation difficile. La chlorpromazine dans les années 1950 en Belgique
}

Benoît Majerus

\section{Summary}

Through a Belgian case study the article tries to trace the gradual stabilisation of chlorpromazine as an antipsychotic in the 1950s. By varying ranges and angles of approach it shows the heterogeneity of actors involved and the semantic bricolage that accompany the marketing of the first antipsychotic. Far from being a revolution, the presence of Largactil ${ }^{\circledR}$ in psychiatric practice is rather characterised by integration into a wider range of medicines and sinuous searching to give sense to this new drug.

Keywords: psychiatry; chlorpromazine; neuroleptic; standardisation;Belgium

\section{Résumé}

A travers le cas d'étude belge, l'article essaie de retracer la lente stabilisation de la chlorpromazine en tant que neuroleptique dans les années 1950. En variant les échelles et les angles d'approche, il s'agit de montrer l'hétérogénéité des acteurs qui interviennent et le «bricolage » sémantique qui accompagnent la mise sur le marché du premier neuroleptique. Loin de constituer une rupture, la présence du Largactil ${ }^{\circledR}$ dans la pratique psychiatrique se caractérise plutôt par une inscription dans un arsenal thérapeutique plus large et par une recherche sinueuse du sens à donner à ce nouveau médicament.

Benoît Majerus, 16E, Rue des Commerçants, B-1000 Bruxelles (bmajerus@ulb.ac.be). 
«Je m'excuse [pour cette lettre peu structurée] ils m'ont donné je devrais dire gavé de 〈Largasil〉 [Largactil ${ }^{\circledR}$ ], infect pilule, qu'il me rend deux idées difficiles à faire suivre mais, comme tu es médecin, j'espère que tu pourras intervenir et demander des explications aux docteurs de l'hôpital.» ${ }^{1}$ C'est sans grand enthousiasme que Christian D., un patient de l'Institut de Psychiatrie de l'Université libre de Bruxelles, raconte les effets du Largactil ${ }^{\circledR}$, le premier neuroleptique à faire son entrée dans le champ psychiatrique au début des années 1950. Or, l'histoire des traitements thérapeutiques en psychiatrie, au $\mathrm{XX}^{\mathrm{e}}$ siècle, est souvent divisée en un avant et un après 1952, moment où trois médecins parisiens présentent les premiers résultats de l'utilisation de la chlorpromazine en tant que médicament psychiatrique ${ }^{2}$. Cette découverte a déjà été souvent racontée soit par les acteurs de la découverte ${ }^{3}$, soit par des médecins s'intéressant à l'histoire ${ }^{4}$, soit par les sciences humaines ${ }^{5}$. Elle est souvent présentée comme ayant provoqué une rupture profonde dans la pratique psychiatrique.

Mais le plus souvent, la narration s'arrête en 1952: une fois le médicament «découvert», sa place dans la thérapie psychiatrique ne semble plus poser question. Depuis plusieurs années, différents chercheurs ont néanmoins souligné qu'au début de sa trajectoire, un nouveau médicament est rarement topographié clairement et qu'il parcourt souvent un chemin sinueux avant d'être stabilisé comme spécifique pour tel ou tel diagnostic ${ }^{6}$. C'est dans cette réflexion que s'inscrit la présente étude qui se penchera sur l'accueil de la chlorpromazine en Belgique. A travers différents points de vue, à partir d'échelles variées ${ }^{7}$, la lente et hésitante inscription de la chlorpromazine en psychiatrie est décrite.

1 HPIB, AS, n¹3515, lettre de Christian D. à un ami (23 juillet 1958). L'orthographe de la lettre a été maintenue. L'impression d'un retard des capacités mentales ne peut pas être réduite au délire des patients. Exactement le même jour, le psychiatre note: «Sa pensée est lente (Largactil).» Christian D. est soumis à ce moment à une dose particulièrement élevée: $100 \mathrm{mg}$ de Largactil $^{\circledR}$ par jour, alors que la plupart des patients reçoivent à ce moment trois doses de $25 \mathrm{mg}$ par jour.

2 Delay/Deniker/Harl 1952: La phénothiazine est «découverte» à la fin du XIXe siècle en Allemagne. Pendant les cinq décennies suivantes, ses usages en médecine seront multiples. Un premier essai psychiatrique en 1899 montre des bons résultats, mais n'arrive pas à attirer l'attention du champ psychiatrique. A partir de la deuxième moitié des années 1940, la substance est utilisée comme sédatif en chirurgie. Au début des années 1950, la phénothiazine est alors transférée sous le nom de chlorpromazine avec beaucoup de succès en psychiatrie.

3 Deniker 1975.

4 Olié/Lôo 1989.

5 Healy 2002.

6 Geest/Whyte/Hardon 1996; Pieters 2005; Greene 2007.

7 Revel 1996. Sur l'importance des jeux d'échelles pour une histoire du médicament: Balz/Hess 2009. 


\section{Vendre le Largactil ${ }^{\circledR}$}

«Découvert» dans les laboratoires de Rhône-Poulenc, le Largactil ${ }^{\circledR}$ est distribué en Belgique par Spécia, le bras pharmaceutique de la firme française. Dès la fin de l'année 1951, celle-ci distribue quelques échantillons à des médecins belges, apparemment sans résultat concret. Dix-huit mois plus tard, Spécia lance une campagne publicitaire pour le marché belge sur lequel elle est jusque-là peu visible, si ce n'est via le Phenergan ${ }^{\circledR}$, un antihistaminique. L'introduction du Largactil ${ }^{\circledR}$ est l'occasion pour l'entreprise française de lancer une campagne de publicité large aussi bien dans des revues médicales généralistes comme Ars medici, la Revue médicale de Louvain ou Le Scalpel que dans la revue psychiatrique belge par excellence, les Acta Neurologica et Psychiatrica Belgica (ANPB). L'effort est donc conséquent pour Spécia, mais reste modeste par rapport à d'autres campagnes publicitaires qui se trouvent à ce moment dans la presse médicale belge. Les annonces sont en noir et blanc et occupent la plupart du temps qu'une demi-page. Spécia passe quatre annonces différentes dans la presse médicale. La première - celle de 1953 ne s'adresse pas encore particulièrement à la psychiatrie ${ }^{8}$. Comme l'a prévu une notice interne pour les agents de vente, le Largactil ${ }^{\circledR}$ est présenté pour trois champs d'utilisation: la chirurgie, la psychiatrie et l'obstétrique. Le médicament est vendu à ce moment-là sous l'étiquette «neuroplégique» et présenté comme un potentialisateur d'autres médicaments (analgésiques, barbituriques ...). Spécia propose le Largactil ${ }^{\circledR}$ surtout sous forme de comprimés, mais indique en plus petit sur ses annonces qu'il existe également en ampoules. La dose indiquée est de 3 à 6 comprimés de $25 \mathrm{mg}$ en 24 heures (75 mg à $150 \mathrm{mg}$ ). Dans une deuxième annonce, peu utilisée - sauf au début de l'année 1954 -, Spécia met déjà davantage l'accent sur l'application en psychiatrie, seule spécialité médicale des trois à bénéficier d'une description plus détaillée des champs d'application. Le Largactil ${ }^{\circledR}$ n'est plus vendu comme un potentialisateur; la seule étiquette qui le caractérise maintenant est celle de «neuroplégique». Parallèlement, Spécia s'essaie également à une autre présentation. En effet, dans plusieurs revues médicales généralistes, le Largactil $^{\circledR}$ est annoncé comme un médicament avec une indication très large visant la «pathologie médicale». Il y est présenté comme combattant aussi bien les insomnies que l'asthme et les vomissements. Le $4560 \mathrm{RP}$, son nom industriel, est même jugé adapté pour combattre la toxicose chez les nourrissons. Ces messages publicitaires qui s'adressent à des publics très divers illustrent «l'indétermination partielle de l'agent thérapeutique» qui caractérise l'histoire 
du premier neuroleptique ${ }^{9}$. Pendant les deux premières années, aussi bien en Belgique, qu'en France et en Allemagne ${ }^{10}$, les distributeurs de la chlorpromazine n'offrent pas encore de spécification pathogénique de leur nouveau produit. Le produit n'est pas caractérisé comme agissant contre une maladie précise mais plutôt défini en fonction des symptômes qu'il est sensé combattre.

Mais à partir de la deuxième moitié de 1954, Spécia fixe le modèle publicitaire qui restera appliqué en Belgique jusqu'au début des années 1960, fin de la campagne publicitaire pour le Largactil ${ }^{\circledR}$ dans les revues médicales. Le Largactil ${ }^{\circledR}$ est dorénavant vendu comme un médicament réservé à la neuropsychiatrie. Ce dernier type de publicité ne se retrouve d'ailleurs presque plus que dans la revue belge de psychiatrie. Dans la communication vers l'extérieur, Spécia a maintenant stabilisé la chlorpromazine dans une spécialité médicale. A partir de la deuxième moitié de 1954, Spécia fait également valoir sur ses dépliants publicitaires le fait que le Largactil ${ }^{\circledR}$ a été classé dans la catégorie A par le Fonds National d'Assurance Maladie-Invalidité (FNAMI), catégorie la plus favorable pour l'utilisation du médicament car elle assure un remboursement de $70 \%$ du prix public ${ }^{11}$. Vu le prix élevé du Largactil ${ }^{\circledR}$, au moins à ses débuts ${ }^{12}$, il s'agit là d'un argument non négligeable.

\section{Le Largactil ${ }^{\circledR}$ dans le discours médical}

Le premier article paraissant en Belgique sur le Largactil ${ }^{\circledR}$ n'est pas publié par une revue à caractère scientifique mais dans une publication éditée par les frères de la Charité, une congrégation spécialisée en psychiatrie et qui dispose aussi d'asiles aux Pays-Bas, en Angleterre, au Canada et sur le continent africain. Ziekenverpleging est une revue destinée aux médecins et aux soignants travaillant pour les frères et contient essentiellement des articles de vulgarisation qui décrivent souvent de très près le quotidien des hôpitaux. Y écrivent aussi bien des médecins que des frères qui travaillent comme infirmiers. C'est l'un de ces derniers qui publie en octobre 1953 - donc relativement tôt - ses premières expériences avec ce nouveau médicament. L'article propose d'utiliser le Largactil ${ }^{\circledR}$ dans deux cas de figure:un usage exclusif pour les psychoses (dosage proposé: $150 \mathrm{mg}$ ) ou un potentialisateur pour des cures de sommeil (dosage variable selon les réactions du patient: entre 50 et

9 Bonah/Massat-Bourrat 2005, 45.

10 Pour la France: Bonah/Massat-Bourrat 2005; pour l'Allemagne: Balz 2009.

11 Fonds National d'Assurance Maladie-Invalidité 1955, 71.

12 De Busscher 1956, 1015. 
$125 \mathrm{mg}$ ). Dans ce premier article, le bilan reste ambigu: «Dans un premier temps, la cure de Largactil n'est pas plus impressionnante que la cure d'insuline. En fait c'est néanmoins le cas: mais elle comporte aussi de nombreux éléments ennuyeux.» ${ }^{13}$ Contrairement à la cure d'insuline, l'attention accordée aux patients (température, pression sanguine ...) doit être continue. Ensuite, l'auteur rapporte également des problèmes cutanés pour les soignants qui utilisent le Largactil ${ }^{\circledR}$ et conseille fortement l'utilisation de gants.

Par la suite, l'accueil est néanmoins plutôt positif dans cette revue qui se caractérise par son approche pratique. Plusieurs auteurs soulignent les changements importants qui peuvent être observés dans les salles. Le rapport général de la congrégation pour l'année 1953 se conclut ainsi: «La cure au Largactil produit surtout des résultats chez des personnes réveillées et agitées, et va peut-être changer à leur avantage l'image des sections où se retrouvent les agités.» ${ }^{14}$ L'accueil chez les frères de la Charité est d'une manière générale positif. En 1954, le médecin chef d'un de leurs hôpitaux les plus importants publie une étude plus large et divise les patients en deux groupes: ceux qui sont incurables mais dont il veut limiter l'agitation, et ceux souffrant de psychoses récentes. Ses conclusions sont positives pour les deux groupes: «Dans un pavillon de patients agités [...], la cure au Largactil crée une atmosphère sereine de calme et de repos. En ce qui concerne les psychoses récentes la cure de Largactil est un outil qui produit rapidement des effets et qui est facile à utiliser.» ${ }^{15} \mathrm{Il}$ souligne que le Largactil ${ }^{\circledR}$ peut être appliqué à un large éventail de patients et qu'à ce niveau le nom - de «large» et «activité» - est jugé justifié. Un bémol dans cet accueil positif: l'auteur utilise le terme de «leucotomie médicamenteuse». Le médicament aurait donc des effets similaires à la lobotomie. Sans être rejetée dans les milieux catholiques, cette méthode y est fortement contestée. Néanmoins Ziekenverpleging reste un défenseur de l'utilisation du Largactil ${ }^{\circledR}$. Encore en 1963, dans un double article où les réflexions critiques ne sont certes pas absentes, le Largactil ${ }^{\circledR}$ est présenté sous un regard positif ${ }^{16}$.

Dans le reste de la presse médicale belge, les contributions se caractérisent très tôt par une certaine méfiance. En 1955, la première étude est publiée en dehors de Ziekenverpleging. Le Belgisch Tijdschrift voor Genees-

13 «Op het eerste gezicht ziet er een largactilkuur zo indrukwekkend niet uit als de insulinekuur. Feitelijk is het zo; maar toch heeft ze haar vervelende kanten», Anonyme 1953, 26.

14 «De largactilkuur [...] boekte vooral bij opgewekten en onrustigen buitengewoon flinke uitslagen, en zal wellicht het beeld van onze onrustige afdelingen voordelig wijzigen», B. D. 1955, 10.

15 «In een paviljoen van onrustige patiënten [...] schept ze [de largactilkuur] een serene atmosfeer van kalmte en rust. Wat betref geneesbare recente psychosen, is de largactilkuur een kostbaar, snel werkend en gemakkelijk hanteerbaar hulpmiddel», Jacobs 1954, 27.

16 Van der Spek 1963. 
kunde correspond davantage à l'image classique d'une revue scientifique. Les auteurs travaillent à l'Université de Gand et ont réalisé une étude sur deux populations psychiatriques importantes: les maniaco-dépressifs et les psychotiques schizophrènes. En ce qui concerne la première catégorie de personnes, le bilan est très clair: «L'efficacité du neuroplégique 4560 R.P. est très largement inférieure aux thérapies électriques.» ${ }^{17}$ En ce qui concerne le deuxième groupe les conclusions sont légèrement plus positives. Les deux auteurs plaident en faveur du Largactil ${ }^{\circledR}$... faute de mieux. Par la suite, la discussion en Belgique reste marquée par l'équipe de l'Université de Gand qui est presque la seule à publier sur ces nouveaux médicaments psychiatriques. Or, le directeur du département de psychiatrie, Jacques De Busscher, est pour le moins sceptique face au Largactil ${ }^{\circledR}$ en particulier, et il le restera face aux neuroleptiques en général.

Les publications belges s'inscrivent bien sûr dans une discussion internationale. Dès 1953, des articles sur la chlorpromazine sont publiés en dehors de la France, en Suisse, en Italie, en Allemagne et en Amérique du Nord. Le Colloque international sur la chlorpromazine et les médicaments neuroleptiques en thérapeutique psychiatrique qui se tient en octobre 1955 à Sainte Anne à Paris ${ }^{18}$, sert de lieu d'échanges où les organisateurs, Delay et Deniker, espèrent stabiliser la chlorpromazine comme médicament psychiatrique. De Busscher y est une des rares voix qui restent très prudentes. Pour lui, le Largactil ${ }^{\circledR}$ est surtout intéressant pour les «malades d'asiles» à cause des «propriétés sédatives», mais la chlorpromazine «ne produit pas la guérison». Il souligne en même temps un autre biais de la plupart des études. Selon De Busscher, qui est également psychanalyste de formation, de nombreux cas de guérison sont dus à «l'influence suggestive exercée par l'offre d'un traitement». En absence «d'un groupe témoin traité à l'aide de comprimés d'aspect, de coloration, et d'emballage identiques», les affirmations reposent, selon le médecin gantois, sur des pieds d'argile ${ }^{19}$. Dans les années qui suivent, les membres de l'équipe gantoise continuent à accompagner d'une manière assez critique la généralisation du Largactil ${ }^{\circledR}$. S'ils reconnaissent une certaine utilité au médicament en ce qui concerne les asiles psychiatriques, ils se montrent sceptiques quant à son utilité générale. Ainsi les effets secondaires sont l'objet d'un article particulier, à la fin duquel les auteurs déconseillent l'utilisation du Largactil ${ }^{\circledR}$ en ambulatoire. A part l'action sédative, ils ne voient

17 «Efficiëntie van het neuroplegicum 4560 R. P. ligt dus zeer diep onder deze van de electrische behandelingsmethode», Evrard/Vandierendonck 1957.

18 Une vision édulcorée sur ce colloque: Lempérière/Robert 2008.

19 De Busscher 1956. Dans une autre contribution de ce colloque, le psychiatre anglais MayerGross souligne également les faiblesses méthodologiques des résultats publiés. 
d'effets ni pour les maniaco-dépressifs, ni pour les neuroses; et même en ce qui concerne les psychoses, l'action du Largactil ${ }^{\circledR}$ ne serait guère convaincante ${ }^{20}$.

Une des lignes de partage potentielles entre les articles qui accueillent la chlorpromazine d'une manière plutôt positive et ceux qui contestent son utilité est le point de vue des auteurs. Pour reprendre la distinction de Joel Braslow entre effectiveness, basée sur un fonctionnement clinique local, et efficacy, basée sur la performance lors de tests cliniques ${ }^{21}$, les auteurs qui travaillent essentiellement dans des structures asilaires soulignent l'utilité immédiate dans leur pratique psychiatrique:l'effet sédatif est d'une certaine manière jugé auto-suffisant. Les autres auteurs sont certes encore loin des essais cliniques randomisés en double aveugle mais ils s'inscrivent néanmoins dans une volonté de standardisation et de scientificité plus avancée. Leur observation porte beaucoup moins sur une population asilaire mais davantage sur des patients ambulatoires. Leur bilan est souvent plus ambigu.

Ce récit jusque-là déjà assez sinueux se complique encore davantage si on quitte cette lecture focalisée sur la psychiatrie. En effet, la chlorpromazine n'est pas pensée dès ses débuts comme un médicament psychiatrique. Le dépouillement systématique d'Ars medici laisse apparaître une autre chronologie et d'autres applications ${ }^{22}$. Entre 1954 et 1956, neuf notices parlent de la chlorpromazine mais seulement trois dans un contexte psychiatrique, les autres abordent des sujets très divers: de l'obstétrique en passant par l'asthme jusqu'à l'otologie. Ce n'est qu'en 1957 que le Largactil ${ }^{\circledR}$ y est exclusivement catégorisé comme médicament psychiatrique. De même, Archiva Medica Belgica, le journal-coupole de toutes les sociétés médicales belges, parle à plusieurs reprises de la chlorpromazine dans les années 1950, mais jamais dans le contexte de la psychiatrie.

\section{Le Largactil ${ }^{\circledR}$ dans la pratique psychiatrique ${ }^{23}$}

L'hétérogénéité du discours médical publié indique déjà une pratique pour le moins variée. L'introduction du Largactil ${ }^{\circledR}$ - durant la première moitié des années 1950 - se fait à un moment où la psychiatrie dispose d'un arsenal mé-

20 Evrard/Vandierendonck 1957, 47.

21 Braslow 1997.

22 Ars medici est le journal qui compte de loin le plus d'abonnés parmi les médecins en Belgique. Ayant comme sous-titre «Revue internationale pour le médecin praticien», il s'agit d'une publication qui comporte quelques articles originaux, mais surtout des résumés d'articles ayant paru dans d'autres revues médicales.

23 Braunschweig 2007. 
dicamenteux et thérapeutique assez large. Depuis la fin du XIX ${ }^{e}$ siècle, différentes substances visant à calmer l'agitation des patients se trouvent sur le marché. Que ce soit l'hyoscine, un alcaloïde isolé chimiquement dans les années 1890, ou l'amytal, un barbiturique mis sur le marché par Lilly dans les années 1920, les médicaments sont utilisés quotidiennement dans la pratique psychiatrique ${ }^{24}$. Ainsi, à l'Institut de Psychiatrie de l'hôpital Brugmann - l'hôpital universitaire de Bruxelles -, plus de la moitié des patients se voient administrer de tels médicaments entre 1931 et 1950. L'utilisation se fait néanmoins dans une approche multi-thérapeutique. En effet, le médicament est rarement administré tout seul: le plus souvent, d'autres outils sont également utilisés. Si différentes formes de balnéothérapie sont d'usage dans les deux premières décennies, les années trente voient apparaître une nouvelle panoplie de thérapies de chocs (cardiazol, insuline, électrochoc ...). Médicaments et thérapies de chocs sont le plus souvent utilisés parallèlement. Et leur fonction dans les asiles est multiple: ils sont à la fois thérapeutiques, sédatifs et disciplinaires, trois niveaux qui ne sont d'ailleurs pas nécessairement séparés dans la pratique psychiatrique.

Il est bien sûr impossible, vu l'échelle choisie, de présenter un aperçu global et systématique de l'introduction du Largactil ${ }^{\circledR}$ dans les hôpitaux en Belgique. Afin de diversifier les angles, l'étude se base d'une part sur les dossiers de patient ${ }^{25}$ de l'Institut de Psychiatrie à Bruxelles et d'autre part sur les dossiers administratifs des frères de la Charité, congrégation religieuse qui gère dans les années 1950 environ un tiers des lits psychiatriques du pays. Exception faite des échantillons envoyés par Spécia en 1951-1952, le Largactil ${ }^{\circledR}$ ne fait son entrée dans les hôpitaux psychiatriques de Belgique qu'au printempsété $1953^{26}$.

A l'Institut de Psychiatrie, département universitaire d'une centaine de lits, la chlorpromazine connaît une introduction rapide. Les psychiatres qui y travaillent ne montrent guère d'intérêt dans leurs publications pour les nouvelles avancées de la psychiatrie biologique, ce qui ne les empêche nullement de recourir à ce nouveau médicament. Sans devenir une formule miracle, le Largactil ${ }^{\circledR}$ change en quelques années la pratique thérapeutique à l'intérieur de l'Institut (tab.1). Il remplace les électrochocs qui étaient un traitement administré à près de la moitié des patients dans les cinq années précédant l'in-

24 Pieters/Snelders 2005.

25 Sur l'utilisation des dossiers de patients en tant que source historique, voir Brändli/ Lüthi/Spuhler 2009.

26 La situation dans les colonies belges ne pourra pas être abordée dans le cadre de cette contribution. Il semble cependant que l'introduction du Largactil ${ }^{\circledR}$ s'y fait au même moment qu'en Belgique. A l'hôpital d'Usumbura (aujourd'hui Bujumbura/Burundi), le Largactil ${ }^{\circledR}$ est administré dès 1953: Vyncke 1957. 
Tab. 1. Evolution des thérapies à l'Institut de Psychiatrie de l'hôpital Brugmann entre 1953 et $1957^{1}$.

\begin{tabular}{llllcc}
\hline & $\mathbf{1 9 5 3}(\mathbf{n}=\mathbf{1 8})$ & $\mathbf{1 9 5 4}(\mathbf{n}=\mathbf{5 9})$ & $\mathbf{1 9 5 5}(\mathbf{n}=\mathbf{5 2})$ & $\mathbf{1 9 5 6}(\mathbf{n}=\mathbf{3 6})$ & $\mathbf{1 9 5 7}(\mathbf{n}=\mathbf{3 6})$ \\
\hline ECT & $33 \%$ & $12 \%$ & $12 \%$ & $9 \%$ & $11 \%$ \\
\hline chlorpromazine & $11 \%$ & $24 \%$ & $29 \%$ & $34 \%$ & $42 \%$ \\
\hline thérapies à l'insuline & $17 \%$ & $15 \%$ & $2 \%$ & $0 \%$ & $3 \%$ \\
\hline autres médicaments & $61 \%$ & $59 \%$ & $85 \%$ & $63 \%$ & $75 \%$ \\
\hline
\end{tabular}

1 Les chiffres de l'Institut de Psychiatrie proviennent d'une banque de données qui repose sur un échantillon (chaque quinzième dossier) des 35000 dossiers de patients qui y sont conservés. Cette banque de données a été construite avec les étudiants de deuxième cycle d'études du séminaire d'histoire 2007-2008 et 2008-2009 à l'Université libre de Bruxelles, séminaire organisé avec Pieter Lagrou.

troduction de la chlorpromazine. D'une manière générale, le Largactil ${ }^{\circledR}$ détrône dans un intervalle de cinq ans la première génération des thérapies biologiques. Leur part dans le traitement, de $60 \%$ au début des années cinquante, tombe à $10 \%$ à la fin de cette décennie. L'utilisation large du Largactil ${ }^{\circledR}$ s'explique également par l'apparent flou du profil thérapeutique. Il est cependant loin d'être considéré comme suffisant. Souvent, son utilisation est combinée avec d'autres thérapies biologiques ${ }^{27}(18 \%$ en combinaison avec des électrochocs) et/ou d'autres médicaments. Seul un dixième de la population ayant reçu du Largactil ${ }^{\circledR}$ n'a pas été soumis à d'autres traitements.

Le champ des diagnostics pour lesquels les psychiatres de l'Institut le prescrivent est très large et ne correspond pas encore au profil thérapeutique des neuroleptiques dans les années 1960 et 1970, quand ceux-ci sont définis comme des médicaments essentiellement antipsychotiques. Dans les années 1950, parmi ceux qui se voient administrer du Largactil ${ }^{\circledast}, 13 \%$ portent le diagnostic d'«éthylisme», $10 \%$ de «dépression» et $5 \%$ de «schizophrénie». Contrairement aux thérapies biologiques comme par exemple les électrochocs, le Largactil ${ }^{\circledast}$ n'est pas une thérapie générée à l'Institut: la différence entre hommes $(53 \%)$ et femmes $(47 \%)$ n'est pas significative ${ }^{28}$.

Dans les asiles des frères de la Charité, la chlorpromazine est également utilisée pour la première fois en 1953. A la fin de l'année, seulement trois des neufs hôpitaux des frères de la Charité comptent le nouveau médicament dans

27 Une évolution similaire peut être observée pour le Burghölzli à Zurich, un des seuls hôpitaux en Europe pour lequel une analyse détaillée quant aux thérapies biologiques au XX ${ }^{\mathrm{e}}$ siècle existe: Meier et al. 2007, 106sq.

28 A part Balz 2009 qui constate une surreprésentation féminine, les autres études (Meier et al. 2007; Beddies/Dörries 1999) notent une surreprésentation masculine parmi les premiers patients à recevoir le Largactil ${ }^{\circledR}$. 
leur arsenal thérapeutique ${ }^{29}$. La relative rupture qui se dégage à l'Institut de Psychiatrie au niveau des thérapies est beaucoup moins prononcée chez les frères de la Charité. Pour 1954, leur revue publie le rapport thérapeutique suivant: 1 lobotomie, 116 cures d'insuline, 354 électrochocs, 13 thérapies de malaria, 285 thérapies au Largactil ${ }^{\circledR}$ et 104 au Serpasil ${ }^{\circledR}$ (réserpine). En ce qui concerne ces deux derniers médicaments, des différences parfois importantes existent également entre les hôpitaux. Un tiers des thérapies au Largactil ${ }^{\circledR}$ sont administrées dans une des neuf institutions gérées par la congrégation. Dans un autre hôpital, le Largactil ${ }^{\circledR}$ n'est pas apprécié et les médecins n'administrent que du Serpasi ${ }^{\circledR 30}$. Mis sur le marché par Ciba en 1953, la réserpine a été largement oubliée par après. Dans les années 1950, des résultats encourageants et son moindre coût expliquent pourquoi elle bénéficie d'un certain intérêt ${ }^{31}$. Jusqu'à la fin des années 1950, la part du Largactil ${ }^{\circledR}$ et de la réserpine augmente mais d'une manière moins importante qu'à l'Institut. En 1958, un cinquième des patients soumis à un traitement dans les asiles des frères de la Charité subissent encore des électrochocs $\left(45 \% \text { une cure de } \text { Largactil }^{\circledR}\right)^{32}$. Le Centre Neuro-Psychiatrique, le plus grand hôpital psychiatrique dans la capitale belge, pratique une toute autre politique. Un membre de la famille Titeca auquel appartient cet hôpital explique en 2000:

Le Largactil était peu employé. Cela peut s'expliquer d'une certaine manière. À l'Institut, on a toujours eu des patients profondément enfoncés dans la psychose. Le Largactil a été utilisé par Delay et Deniker probablement sur des patients moins détériorés sur le plan psychotique. Moi j'ai toujours eu l'impression que le Largactil n'était guère efficace, que c'était de l'eau de robinet. ${ }^{33}$

Cette approche quantitative de l'utilisation du Largactil ${ }^{\circledR}$ dans quelques hôpitaux permet de formuler quelques hypothèses. A moyen terme, le Largactil ${ }^{\circledR}$ semble surtout faire diminuer la part des thérapies de choc, mais ce processus n'est ni très rapide ni uniforme. L'entrée de la chlorpromazine dans l'arsenal thérapeutique se fait selon des routines thérapeutiques existantes. Son profil thérapeutique flou des débuts, proche des sédatifs plus classiques, explique sans aucun doute son intégration progressive dans la pratique psychiatrique. Comme pour les médicaments pré-modernes, la chlorpromazine est pensée comme un outil complémentaire et non exclusif par rapport aux autres thérapies existantes. A la fin de la décennie, les neuroleptiques sont encore loin d'occuper une place hégémonique dans la thérapie psychiatrique.

29 B. D. $1954,10$.

30 B. D. 1955.

31 Healy 2002, 101-106.

32 Archives du Provincialraad - Broeders van Liefde (Gent), 7. Apostolaat: Verpleging en psychiatrie algemeen - 3, Overzicht van de behandelingen 1958.

33 Missa 2006, 309. 


\section{Un acteur oublié}

L'histoire des médicaments reste souvent enfermée dans un binôme firmes pharmaceutiques (laboratoire, vente, publicité ...) vs hôpitaux (médecins, patients ... $)^{34}$. Au moins pour l'après-1945, un acteur important y manque: le Welfare State et ses multiples organisations (para-)étatiques ${ }^{35}$. Dans le cas belge, le FNAMI joue un rôle central. Fondé en 1945, cet organisme sert d'interface entre mutualités, hôpitaux et patients et décide notamment des remboursements (partiels ou complets) des prestations médicales et des médicaments. A ce niveau se (re)jouent donc de nombreuses questions qui ont été abordées auparavant pour les autres échelles: rupture ou non, classification, standardisation ... Nous avons déjà signalé que Spécia utilise la reconnaissance par la FNAMI comme outil de vente. Les conditions d'admission au processus d'évaluation sont les suivantes: disposer d'un échantillon de «vente normal» et (avoir demandé) un contrôle de laboratoire afin de déterminer la composition du médicament ${ }^{36}$. A ce moment-là, aucune preuve ne doit encore être fournie quant aux effets thérapeutiques et/ou secondaires ${ }^{37}$.

Une première demande pour le Largactil ${ }^{\circledR}$ est rejetée en avril 1953 par le Conseil Technique Pharmaceutique de la FNAMI à cause de l'absence du contrôle de laboratoire. A peine un mois plus tard, Spécia réintroduit une demande, accompagnée cette fois d'une demande d'analyse, et, en été 1953, la chlorpromazine est inscrite dans le Tarif Officiel: le médicament devient donc remboursable, condition sine qua non pour une utilisation importante à l'intérieur des hôpitaux. Le Largactil ${ }^{\circledR}$ est classé en 1953 dans la catégorie VIII.5., qui est celle des anti-parkinsoniens. Les raisons de cette classification sont probablement d'ordre chimique (chlorpromazine et anti-parkinsoniens appartenant à la classe des phénotiazines). La logique chimique est déterminante, ce qui n'a rien d'étonnant vu la composition de la commission, où siègent majoritairement des pharmaciens. Cette classification basée sur la parenté chimique s'avère néanmoins peu fonctionnelle: en effet on y trouve des spécialités qui «ne sont pas des antiparkinsoniens, mais peuvent au contraire, provoquer le parkinsonisme comme réaction secondaire», ainsi que le constate le Conseil Technique des Spécialités Pharmaceutiques ${ }^{38}$. Pendant deux ans, les différentes commis-

34 Gaudillière 2007.

35 Kaelble 2007, 332-360.

36 Archives de l'INAMI (A-INAMI), Procès-verbaux du Conseil Technique Pharmaceutique, séance du 3 avril 1953.

37 Ce n'est qu'à partir de 1960 que l'admission des médicaments est davantage formalisée, en particulier avec l'obligation d'effectuer des expérimentations pharmacologiques, cliniques, toxicologiques et d'accoutumance.

38 Archives de l'INAMI (A-INAMI), Note n¹46 du Conseil Technique des Spécialités Pharmaceutiques (non daté, probablement décembre 1958). 
sions discutent donc de la nature exacte de ce nouveau médicament afin de savoir comme réorganiser la catégorisation de la pharmacopée. Finalement, une nouvelle catégorie est créée en 1960: les neuroplégiques à action antipsychotique. En se basant sur la classification d'un psychiatre suisse, Felix Labhardt ${ }^{39}$, le Conseil Technique des Spécialités Pharmaceutiques distingue désormais deux catégories: les neuroleptiques et les tranquillisants. La première catégorie est définitivement inscrite dans le cadre psychiatrique qui devient maintenant une condition de remboursement. Au moins jusqu'à la fin des années 1960, le Largactil ${ }^{\circledR}$ sera le médicament standard en ce qui concerne les neuroleptiques pour la FNAMI. C'est lui qui sert de référence pour déterminer les prix de nouveaux neuroleptiques, pour en mesurer les effets.

\section{Au-delà du Largactil ${ }^{\circledR}$}

L'introduction des neuroleptiques offre une nouvelle légitimité à la psychiatrie comme discipline. Souvent marginalisée dans le champ médical, la psychiatrie bénéficie d'une certaine valorisation grâce aux neuroleptiques. A côté des antibiotiques et stéroïdes, ces nouvelles substances psychiatriques suscitent une nouvelle vague d'espoir thérapeutique après la Deuxième Guerre mondiale ${ }^{40}$. La thérapie psychiatrique devient d'ailleurs un sujet sur lequel les revues psychiatriques publient à nouveau des articles à partir des années 1950, alors que le sujet n'était guère présent dans la décennie précédente (tab. 2).

Tab. 2. Articles «thérapeutiques» dans les Acta (1950-1960)ํ․

\begin{tabular}{lrrrrrrrrrrr}
\hline & $\mathbf{1 9 5 0}$ & $\mathbf{1 9 5 1}$ & $\mathbf{1 9 5 2}$ & $\mathbf{1 9 5 3}$ & $\mathbf{1 9 5 4}$ & $\mathbf{1 9 5 5}$ & $\mathbf{1 9 5 6}$ & $\mathbf{1 9 5 7}$ & $\mathbf{1 9 5 8}$ & $\mathbf{1 9 5 9}$ & $\mathbf{1 9 6 0}$ \\
\hline total-articles & 26 & 58 & 48 & 55 & 63 & 63 & 46 & 60 & 67 & 71 & 78 \\
\hline psychotropes & 0 & 0 & 0 & 0 & 0 & 1 & 1 & 2 & 4 & 7 & 26 \\
\hline ECT & 0 & 1 & 1 & 1 & 0 & 0 & 0 & 0 & 0 & 2 & 1 \\
\hline psychothérapie & 0 & 0 & 0 & 1 & 0 & 0 & 0 & 2 & 0 & 5 & 0 \\
\hline autres & 1 & 0 & 0 & 1 & $8^{2}$ & 1 & 0 & 0 & 1 & 0 & 0 \\
\hline
\end{tabular}

1 Par «articles thérapeutiques», j'entends les articles consacrés spécifiquement à la discussion d'une thérapie distinctive, à l'exception des articles consacrés à l'épilepsie.

2 Numéro consacré à la psychochirurgie.

Si les neuroleptiques offrent donc de nouvelles perspectives à la psychiatrie à l'intérieur de la discipline, ils contribuent également à une valori-

39 Felix Labhardt a joué un rôle important dans l'introduction de la chlorpromazine en Suisse. Il est un des organisateurs du premier colloque sur le médicament à Bâle en 1953. Il publiera par la suite de nombreux articles sur les neuroleptiques et sera un membre actif du Collegium Internationale Neuro-Psychopharmacologicum. Je remercie Magaly Tornay (Université de Zurich) pour ces informations.

40 Pieters 2005, 182. 
sation dans le champ médical. Peu présente dans les journaux médicaux généralistes avant la première moitié des années 1950, la psychiatrie y fait ensuite une entrée remarquée. Ainsi la Belgisch Tijdschrift voor Geneeskunde publie très régulièrement à partir de 1953 des articles relatifs à la psychiatrie, tous consacrés à la médication psychoactive.

Une des conditions de ce gain de légitimité est néanmoins de pouvoir nommer correctement le nouveau produit, entreprise qui s'avère extrêmement difficile et qui reste caractérisée encore aujourd'hui par une certaine instabilité. L'introduction du Largactil ${ }^{\circledR}$ conduit à des essais multiples de réorganisation classificatrice de la pharmacopée. Dans un premier temps, les «inventeurs» du Largactil ${ }^{\circledR}$ le qualifient de «neuroplégique», avant de proposer dès 1955 le terme de «neuroleptique», qui indique l'action du médicament sur le système nerveux central et qui comporte moins la notion de paralysie contenue dans le premier terme. Par la suite, les essais classificatoires se multiplient ${ }^{41}$. Les enjeux de ces débats sémantiques sont multiples: établir la nouveauté du médicament, illustrer la rupture que constitue le Largactil ${ }^{\circledR}$, décrire son action thérapeutique et/ou sédative, définir les propriétés pharmacologiques, voire réorganiser la nosographie psychiatrique ${ }^{42}$.

Paul Sivadon, professeur de psychiatrie à l'Université libre de Bruxelles et conseiller de l'Organisation Mondiale de la Santé, propose dans son cours de psychiatrie en 1960 la division suivante: «tranquillisants», n'ayant aucun effet thérapeutique dans les psychoses, mais utiles dans des cas d'anxiété, «neuroleptiques», «véritables médicaments» qui entraînent souvent la guérison dans les psychoses, «thymo-analeptiques», pour les états dépressifs, et «psychotoniques» ${ }^{43}$. Autour de 1960, plusieurs auteurs belges essaient de normaliser un domaine qui semble de plus en plus chaotique, tant se multiplient les nouveaux médicaments - portant souvent plusieurs noms de commercialisation. A. F. De Schaepdrijver, qui travaille à l'Institut de pharmacologie Heymans à Gand, propose trois catégories: les «psychoanaleptica», les «tranquilizers» et les «psychosomimetica». Dans le premier groupe se trouvent les médicaments qui activent l'activité psychique comme les amphétamines. Dans le deuxième groupe, il place aussi bien les plus anciens barbituriques que les nouveaux Largactil ${ }^{\circledR}$ et Serpasil ${ }^{\circledR}$. Finalement, la dernière catégorie comprend des substances qui peuvent provoquer des psychoses $^{44}$. Deux ans plus tard, le modèle a davantage évolué. Deux catégories sont maintenues, les «psychoanaleptica» et les «psychosomimetica»; les «tranquilizers» sont remplacés par les «psychoplegica»,

41 Donadieu 1963.

42 King/Voruganti 2002.

43 Sivadon 1960, 106.

44 De Schaepdrijver 1959. 
mais le contenu reste inchangé45. La même année, André Simonart de l'Université Catholique de Louvain, dans un article sur les «psychotropiques», distingue trois groupes: les «psycholeptiques», les «psycho-analeptiques» et les «psychodysleptiques». Son critère de catégorisation est l'influence des substances sur l'activité mentale. Ce qui est particulièrement intéressant dans cet exemple est la reconnaissance du fait que, confronté à un «manque de tests objectifs et à la complexité infinie et encore ignorée des fonctions psychiques et nerveuses» des «psychotropiques», le psychiatre doit être conscient du caractère bancal de ces classifications ${ }^{46}$. Même dix ans après la mise sur le marché, la nomenclature de la pharmacopée est loin d'être stabilisée.

\section{Conclusion}

Le cas d'étude belge s'inscrit dans deux débats historiographiques en cours. D'une part, il permet d'interroger la proposition de deux historiens néerlandais Toine Pieters et Stephen Snelders, qui ont suggéré de relire l'histoire des médicaments psychoactifs avec le modèle de Seige ${ }^{47}$. Ce cycle formulé une première fois en 1912 par le psychiatre allemand Max Seige se caractérise par trois étapes: phase ascendante qui s'accompagne d'espoirs importants, phase critique où les déceptions se multiplient et finalement utilisation limitée. L'évolution du Largactil ${ }^{\circledR}$ en Belgique pendant la première décennie ne se laisse cependant guère enfermer dans cet idéal-type. On distingue plutôt des évolutions parallèles qui dépendent des contextes respectifs d'application. Plutôt que de suivre un modèle linéaire, une analyse à plusieurs échelles semble davantage apte à rendre compte de l'évolution du Largactil ${ }^{\circledR}$.

D'autre part, l'exemple choisi interroge aussi les notions de rupture et de révolution souvent avancées dans l'historiographie du Largactil ${ }^{\circledR}$. Si, rétrospectivement, 1952 peut apparaître comme date de naissance du «médicament moderne $\gg^{48}$ en psychiatrie, les années 1950 montrent la lente dynamique de sa catégorisation comme substance thérapeutique en psychiatrie. D'une signification multiple (médicament pour l'obstétrique, la chirurgie, la psychiatrie ...), la chlorpromazine est peu à peu réduite à un domaine. Ce processus est aussi bien visible au niveau du producteur - l'action large du Largactil ${ }^{\circledR}$ dans la publicité - que dans la réflexion scientifique des médecins sur l'utilisation de la chlorpromazine. Souvent présentée après coup comme un médicament miracle, la chlorpromazine n'est guère vécue comme telle dans les années 1950.

45 De Schaepdrijver 1961.

46 Simonart 1961.

47 Snelders/Kaplan/Pieters 2006.

48 Pignarre 1995. 


\section{Bibliographie}

Anonyme, «De largactil in de psychiatrie», Ziekenverpleging (1953) 20-26

B. D., «Zo ging 1953 over onze psychiatrische Inrichtingen», Ziekenverpleging (1954) 7-15

- «Bijzondere Aspecten van de Verpleging van onze Geesteszieken in 1954», Ziekenverpleging (1955) 8-14

Balz, Viola, Zwischen Wirkung und Erfahrung - Psychopharmaka neu denken. Eine historische Analyse der Wirksamkeitskonstruktion der Neuroleptika in der Bundesrepublik Deutschland, 1950-1980, Dissertation, Technische Universität Carolo-Wilhelmina zu Braunschweig (2009)

- /Volker Hess, "Psychopathology and psychopharmacology. Standardisation from the bottom up, using the example of neuroleptics", in: Christian Bonah/Christophe Masutti/Anne Rasmussen/Jonathan Simon (eds), Harmonizing Drugs. Standards in 20th-Century Pharmaceutical History (Paris 2009) 273-296

Beddies, Thomas/Andrea Dörries (Hg.), Die Patienten der Wittenauer Heilstätten in Berlin: 1919-1960 (Husum 1999)

Bonah, Christian/Séverine Massat-Bourrat, «Les «agents thérapeutiques`. Paradoxes et ambiguïtés d'une histoire des remèdes aux $\mathrm{XIX}^{\mathrm{e}}$ et $\mathrm{XX}^{\mathrm{e}}$ siècles», dans: Christian Bonah/Anne Rasmussen (éds), Histoire et médicament aux XIXe et XX $X^{e}$ siècles (Paris 2005) 23-61

Brändli, Sibylle/Barbara Lüthi/Gregor Spuhler (Hg.), Zum Fall machen, zum Fall werden: Wissensproduktion und Patientenerfahrung in Medizin und Psychiatrie des 19. und 20. Jahrhunderts (Frankfurt a. M. 2009)

Braslow, Joel T., Mental Ills and Bodily Cures: Psychiatric Treatment in the First Half of the Twentieth Century (Berkeley 1997)

Braunschweig, Sabine, «Einfluss der Neuroleptica auf den psychiatrischen Pflegealltag», Medizin, Gesellschaft und Geschichte 26 ( 2007) 53-57

De Busscher, Jacques, «Impressions personnelles sur les effets de l'atarax de la chlorpromazine, de la réserpine et du AY 5406-1 (parasan) en thérapeutique psychiatrique», Encéphale 43 (1956) 1007-1023

De Schaepdrijver, A. F., «Over psychofarmacologie», Belgische Tijdschrift voor Geneeskunde 15 (1959) 1132-1155

- «Psychotrope Farmaca», Belgische Tijdschrift voor Geneeskunde 17 (1961) 1041-1071

Delay, Jean/Pierre Deniker/Jean-Marie Harl, «Utilisation en thérapeutique psychiatrique d'un phénothiazine d'action centrale élective (4560 RP)», Annales Médico-Psychologiques 110 (1952) 112-117

Deniker, Pierre, «Qui a inventé les neuroleptiques?», Confrontations psychiatriques 13 (1975) 7-18

Donadieu, Yves, 1952-1962: essai de synthèse d'une décade de thérapeutique psychotrope: (classification et nomenclature des médications chimiothérapeutiques), thèse pour le doctorat en médecine, Faculté de Médecine (Paris 1963)

Evrard,A./R.Vandierendonck, «Een terugblik op 100 met chlorpromazine behandelde gevallen», Belgische Tijdschrift voor Geneeskunde 13 (1957) 43-47

Fonds National d'Assurance Maladie-Invalidité, Rapport général sur l'Année Sociale 1955 $2^{e}$ Partie-Rapport du service médical (Bruxelles 1955)

Gaudillière, Jean-Paul, «L'industrialisation du médicament: une histoire de pratiques entre sciences, techniques, droit et médecine», Gesnerus 64 (2007) 93-108

van der Geest, Sjaak/Susan Reynolds Whyte/Anita Hardon, "The anthropology of pharmaceuticals: a biographical approach", Annual Review of Anthropology 25 (1996) 153-178

Greene, Jeremy, Prescribing by Numbers: Drugs and the Definition of Disease (Baltimore 2007)

Healy, David, The Creation of Psychopharmacology (Cambridge 2002)

Jacobs, R., «Klinische Ervaring met Largactyl te Zelzate», Ziekenverpleging (1954) 15-27

Kaelble, Hartmut, Sozialgeschichte Europas: 1945 bis zur Gegenwart (Munich 2007)

King, Caroline/Lakshmi N. P. Voruganti, "What's in a name? The evolution of the nomenclature of antipsychotic drugs", Journal of Psychiatry \& Neuroscience 27 (2002) 168-175

Lempérière, Thérèse/Roger Robert, «La révolution neuroleptique, le Congrès de 1955», dans: Jacques Arveiller (éd.), Psychiatries dans l'histoire (Caen 2008) 233-240 
Meier, Marietta/Brigitta Bernet/Roswitha Dubach/Urs Germann, Zwang zur Ordnung: Psychiatrie im Kanton Zürich, 1870-1970 (Zürich 2007)

Missa, Jean-Noël, Naissance de la psychiatrie biologique: Histoire des traitements des maladies mentales au XX $X^{e}$ siècle (Paris 2006)

Olié, Jean-Pierre/Henri Lôo, «Jean Delay (1907-1987), compositeur et acteur de la dernière révolution psychiatrique», Psychiatrie française 20 (1989) 87-89

Pieters, Toine, Interferon: The Science and Selling of a Miracle Drug (London 2005)

- /Stephen Snelders, "Mental ills and the 'hidden history' of drug treatment practices", in: Marijke Gijswijt-Hofstra/Harry Oosterhuis/Hugh Freeman (eds), Psychiatric Cultures Compared: Psychiatry and Mental Health Care in the Twentieth Century: Comparisons and Approaches (Amsterdam 2005) 381-401

Pignarre, Philippe, Les deux médecines. Médicaments, psychotropes et suggestion thérapeutique (Paris 1995)

Revel, Jacques (éd.), Jeux d'échelles: la micro-analyse à l'expérience (Paris 1996)

Simonart, André, «Essai de classification des psychotropiques», Revue Médicale de Louvain (1961) 147-152

Sivadon, Paul, Psychiatrie (Bruxelles 1960)

Snelders, Stephen/Charles Kaplan/Toine Pieters, “On cannabis, chloral hydrate, and career cycles of psychotrophic drugs in medicine", Bulletin of the History of Medicine 80 (2006) 95-114

Swazey, Judith P., Chlorpromazine in Psychiatry (Cambridge, Mass. 1974)

Van der Spek, P.A.F., «Largaktil en geestelijke Gezondheid. II», Psychiatrie en Verpleging 4 (1963) 13-16

Vyncke, J., Psychoses et névroses en Afrique centrale (Bruxelles 1957) 